\section{Highlights in Non-Prostate Genitourinary \\ Tumors at ESMO 2021: Revolutionizing \\ Management Options for Better Therapy}

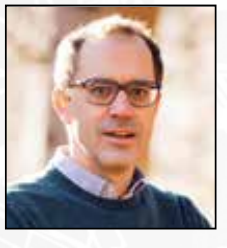

PD Dr Richard Cathomas

Cantonal Hospital of Graubünden
Carion

Chur, Switzerland

Changes in treatment scheduling may lead to clinical benefit in patients with metastatic renal cell carcinoma

STAR is a UK phase II/III multicenter, randomized controlled tria designed to determine if a tyrosine kinase inhibitor (TKI) drugfree interval strategy (DFIS) was non-inferior to a conventional continuation strategy (CCS) in the first-line treatment of were randomized 1:1 to DFIS or CCS. After 24 weeks of sunitinib or pazopanib treatment, DFIS patients took a treatment break, until disease progression, with additional breaks dependent on disease response and patient/clinician choice. In in previously untreated patients with metastatic RCC demonstrated no evidence of excessive tumor growth at discontinuation and presented encouraging survival outcomes ${ }^{2}$

The primary endpoint was overall survival (OS) and it must demonstrate predefined noninferiority $(\leq 7.5 \%)$ in intention-totreat (ITT) and per-protocol (PP) population. ${ }^{1}$ The trial met its endpoint in the ITT population (HR: 0.97 [95\% Cl: 0.83-1.12] but not in PP population (HR: 0.94 [95\% Cl: 0.80-1.09]). In addition, patients on the intermittent schedule did not have negative impact on their quality of life and therefore did not experience symptomatic progression. Furthermore, a substantia financial saving was also observed for patients receiving the intermittent schedule. Although first-line single-agent TKls are no longer the standard of care for patients in this setting, this study suggests that changes to TKI management may be of clinical significance.

The phase II PRISM trial evaluated nivolumab in combination with alternatively scheduled ipilimumab in the first-line treatment of patients with advanced RCC. A total of 192 patients were randomized $1: 2$ to receive 4 doses of ipilimumab once
every 3 weeks (conventional) or once every 12 weeks (modified pilimumab), in combination with nivolumab, until disease progression or unacceptable toxicity. The primary endpoint was the proportion of patients with a grade 3-4 treatment-related adverse event (TRAE) within 12 months of initiating the treatment, while the secondary endpoint included progression-free survival (PFS) at 12 months and objective response rate (ORR). The trial met its primary endpoint as TRAEs were significantly lower amongst patients receiving modified iplimumab compared with conventional ipilimumab (32.8\% v 53.1\%; odds ratio: 0.43 [90\% Cl: 0.25-0.72]; $\mathrm{p}=0.0075)$, corresponding to a reductio of grade $3-4$ adverse events (AEs) by 20.3\%. This reduction was mostly in arthralgia (1.6\% vs $7.8 \%$ ), colitis (3.9\% vs $6.3 \%$ ), ( $.4 \%$. Wheatinine (0\% vs $1.6 \%$ ) and increased lipase (1.6\% vs 9.4\%. While this study was not sufficiently powered to compare the efficacy, the PFS for patients with intermediate and poor-risk Texperiencing an AE are similar, and the same is true for OS. This study highlights that the benefit of TKIs may differ according to their treatment schedule. The benefit for the patients is a decrease in toxicity, leading to an improved quality of life. Patient dherence may also improve, which could impact the efficacy of strestion, further studies are warranted into shedre first-line

Next steps to inpove management of patients with

urothelial carcinoma

The phase II NORSE trial investigated the eficacy and saety of

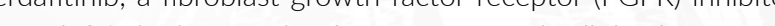
or endanthit plus cetrellnab, a programmed cell death proten 1 (PD-1) Urothelial carcinoma and FGFR alterations." The trial enrolled for FG for FGFR2 or FGR R3 mutations (or fusions). Patients were then pandus cere $1: 1$ to

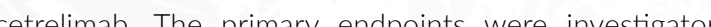
included disease control rate (DCR) time to response (TTR) and duration of response (DOR). In the erdafitinib monotherapy group, an ORR of $33 \%$ was reported compared with $68 \%$ in the cetrelimab/erdafitinib dual therapy group. In addition, $6 \%$ of the patients attained complete remission (CR) in the monotherapy group versus $21 \%$ in the dual therapy group. Regarding side effects, FGFR inhibitors can lead to hyperphosphateming ${ }^{5}$ while PD-1 inhibitors may cause colitis and diarrhea. ${ }^{6}$ In conclusion appears worthwhile to further investigate this treatment regimen, given its promising efficacy.

The VESPER trial was a practice-changing, phase III study conducted in France that investigated the use of neoadjuvant or adjuvant chemotherapy in patients with nonmetastatic muscleInvasive bladder cancer (MIBC).? Patients were randomly assigned to two different chemotherapy regimens: either four cycles of cisplatin gemcitabine (GM/CS) or six cycles of dose(dd-MVAC) repeated every two weeks (with granulocyte-colony stimulating factor [G-CSF] support) before surgery (neoadjuvant group) or after surgery (adjuvant group). The majority of the patients (88\%) in the trial received neoadjuvant treatment and $60 \%$ of these patients received the planned six cycles in the dd-MVAC arm. The pathological response rate was better in the dd-MVAC group with $42 \%$, compared with $36 \%$ for the GM/CIS group. Interestingly, the side effect profile between the two groups was similar; however, the dose-dense MVAC group was supported with G-CSF treatment. Compared with other phase trials, the complete pathological response rate of $42 \%$ in the VESPER trial is among the highest rate achieved. The primary endpoint of PFS was not met for the whole trial population since the adjuvant patients did not benefit as much from dd- MVAC. However, for the neoadjuvant chemotherapy patients, there is a clear and significant difference in favor of dd-MVAC. Taken logether, dd-MVAC cisplatin-based chemotherapy should be considered as a new standard of care for fit patients.

1. Brown JE, Royle KL, Ralph C, et al. LBA28 STAR: A
andomised multi-stage phase IIIlll trial of stand randomised muth-stage phase IIIIII trial of standal
first-line therapy (sunititinib or pazopanib) comparing

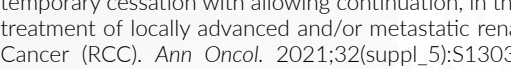

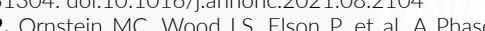
Study of Intermitent Sunitinib in Previously Untreated
Patients With Metastatic Renal Cell Carcinoma. $J$ Cling

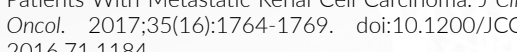
3.Vasudev NS, Ainsworth G, Brown S, et al. LBA29
Nivolumab in combination with alternatively sched

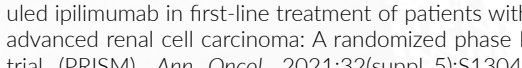

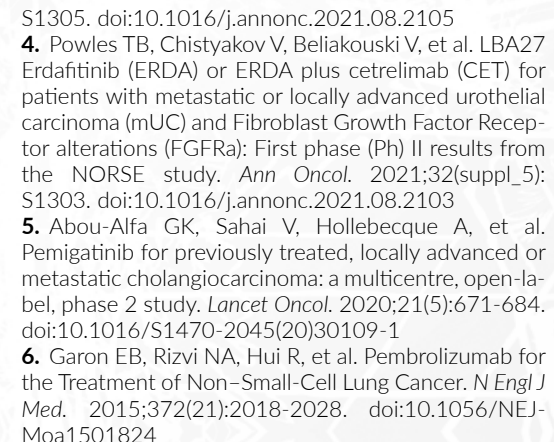

assessed overall response rate (ORR) and safety. The secondary
7. Pfister C, Gravis G, Fléchon A, et all. Randomized

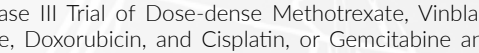

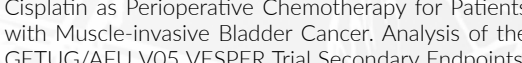
GETUG/AFU VOS VESPER Trial Secondary Endpoints
Chemotherapy Toxicity and Pathological Responses Eur Urol. 2021;79(2):214-221. doi:10.1016/j.euruor 8. Papachristofilou A, Bedke J, Hayoz S, et al. LBA3O
Single-dose carboplatin followed by involved-node radiotherapy as curative treatment for seminoma stage
IIA B B Effccacy results from the international multi- 